\title{
The robustness of glycolysis in yeast to internal and external noise
}

\author{
Eva Gehrmann ${ }^{1}$, Christine Gläßer ${ }^{2}$, Yaochu Jin ${ }^{3}$, Bernhard Sendhoff ${ }^{4}$, Barbara Drossel ${ }^{1}$, and Kay Hamacher ${ }^{5}$ \\ ${ }^{1}$ Institut für Festkörperphysik, TU Darmstadt, \\ Hochschulstraße 6, 64289 Darmstadt, Germany \\ ${ }^{2}$ Helmholtz Zentrum München, \\ German Research Center for Environmental Health, \\ Institute of Bioinformatics and Systems Biology (IBIS), \\ Ingolstädter Landstr. 1, 85764 Neuherberg, Germany \\ 3 Dept. of Computing, University of Surrey, \\ Guildford, Surrey, GU2 7XH, United Kingdom \\ ${ }^{4}$ Honda Research Institute Europe GmbH, \\ Carl-Legien-Straße 30, 63073 Offenbach/Main, Germany \\ ${ }^{5}$ Dept. of Biology and Dept. of Computer Science, \\ TU Darmstadt, Schnittspahnstr. 10, \\ 64287 Darmstadt, Germany
}

\begin{abstract}
Glycolysis is one of the most essentialintra-cellular networks, found in a wide range of organisms. Due to its importance and due to its $\mathrm{m}$. details of this process have been performed. Until now, however, there has been no comprehensive investigation of the robustness of this important process with respect to internal and external noise. To close this gap, we applied two complementary and mutually supporting approaches to a fullscale model of glycolysis in yeast: a) a linear stability analysis based on a generalized modeling that deals only with those effective parameters of the system that are relevant for its stability, and b) a numerical integration of the rate equations in the presence of noise, which accounts for imperfect mixing. The results suggest that the occurrence of metabolite oscillations in part of the parameter space is a side effect of the optimization of the system for maintaining a constant ATP level in the face of a varying energy demand and of fluctuations in the parameters and metabolite concentrations.
\end{abstract}

\section{INTRODUCTION}

The process of glycolysis, considered the paradigm of a classical metabolic process, consists of a series of well studied steps. Nevertheless, the complete pathway of glycolysis still presents important challenges concerning the quantitative aspects of its robustness, its improvability by biotechnological means, and its suitability as a basis for synthetic biology.

Glycolysis produces ATP by degrading glucose, and such a process canno $\equiv$ both maximally fast and efficient at the same timer there is a tradeoff between rate and yield [1-4], with the consequence that organisms with an unlimited availability of resources maximize the rate of ATP production by confining themselves to fermentation, i.e., they perform only glycolysis, while organisms that have limited resources perform respiration, which has a slower rate of ATP production, but a much higher yield per molecule glucose. Stephani et al. [5] searched for signatures of optimization in the structural features of ATP-producing pathways - concluding that the existence of ATP-producing reactions at the end and ATPconsuming reactions at the beginning of the glycolytic pathway is a result of optimization.

Chandra et al. [6], who investigated a simplified model of glycolysis, argue that those features that increase metabolic efficiency and robustness to disturbances, namely a high degree of autocatalysis and a not too fast response to fluctuations, decrease the dynam- ical stability of the process and drive it into sustained oscillations. A. Goldbeter [14] and Sel'kov [15] identified the allosteric enzyme phosphofructokinase (PFK) as the generator of these oscillations, as it is inhibited by the product of glycolysis, ATP. In contrast, Bier et al. [16], using a mathematical core model of glycolysis, found that the oscillation frequency is sensitive to the change of several parameters and conclude that regulation and control need to be attributed to more than one steps.

These observations contributed to the discussion of the last 60 years [7-13], whether the oscillations observed in glycolysis are a side-effect of other, desired network features, or whether they serve a purpose. Even more generally, it is not yet fully understood whether the process of glycolysis optimizes a particular singular characteristic, or whether it has to be understood as a holisticly adapted module - a question at the core of systems biology [17].

Apart from oscillations, random fluctuations in the metabolite concentrations can also cause instabilities. In order to function properly and guarantee the cell's survival, metabolic networks need to be sufficiently robust against noise. Buzi et al. [18] showed that the region of attraction of the fixed point at which the cell operates is large, and that therefore random fluctuations cannot easily push the system outside this region.

While simplified core models of glycolysis provide useful insights, a deeper understanding requires full-scale models. Rizzi et al. [19] investigated a detailed model for glucose transport into the cell in order to understand 
quantitatively the response of the glycolytic pathway to a sudded glucose supply. A detailed model for the entire pathway in Saccharomyces cerevisiae was presented by Hynne et al. [20]. They included all relevant reaction steps into the model and determined the values of those kinetic parameters that have not been measured by fitting the steady state of the model to all available experimental data for Saccharomyces cerevisiae. They found that the point of operation is near a Hopf-bifurcation. This model has since then been used for interpreting experiments and for systematically performing model reductions $[21,22]$.

In this paper, we will use the full-scale model by Hynne et al. [20] in order to perform two mutually supporting types of investigations:

- a linear stability analysis under variation of the parameters and fixed point concentrations, using the generalized method developed by Gross and Feudel [23] and adapted to metabolic networks by Steuer et al. [24], yielding the stability of fixed points and the occurrence of bifurcations in dependence of general features of the system, without the need to specify the steady state or the regulatory functions;

- a numerical integration of the rate equations subject to random noise in the concentrations to mimic non-perfect mixing, varying kinetic parameters and metabolite concentrations, and evaluating fluctuations and correlations in the system.

Our main findings are that the pathway has the property that the stationary ATP level remains constant when glucose supply or ATP demand change and that this behavior is robust in the presence of periodic oscillations and $\theta$ stochastic fluctuations. Furthermore, our results resolve the puzzle of why some studies identified one enzyme as the key player responsible for the oscillations, while others found that several reactions affect the oscillations.

The paper is structured as follows. In Section II, we outline the full-scale model of glycolysis in Saccharomyces cerevisiae, followed by the generalized method and our implementation of noise used for the analysis in Section III. Section IV presents and explains the results of the two approaches. Finally, we discuss our findings in Section V.

\section{FULL-SCALE MODEL OF GLYCOLYSIS IN SACCHAROMYCES CEREVISIAE}

The process of glycolysis degrades glucose into pyruvate in the cytosol of the cell. The essential reactions are shown in Fig. 1. After the transport of glucose (Glc) into the cell (reactions 1 and 2), the enzyme hexokinase (HK) catalyzes phosphorylation of glucose (reaction 3). This process, which moves one phosphate group of adenosine

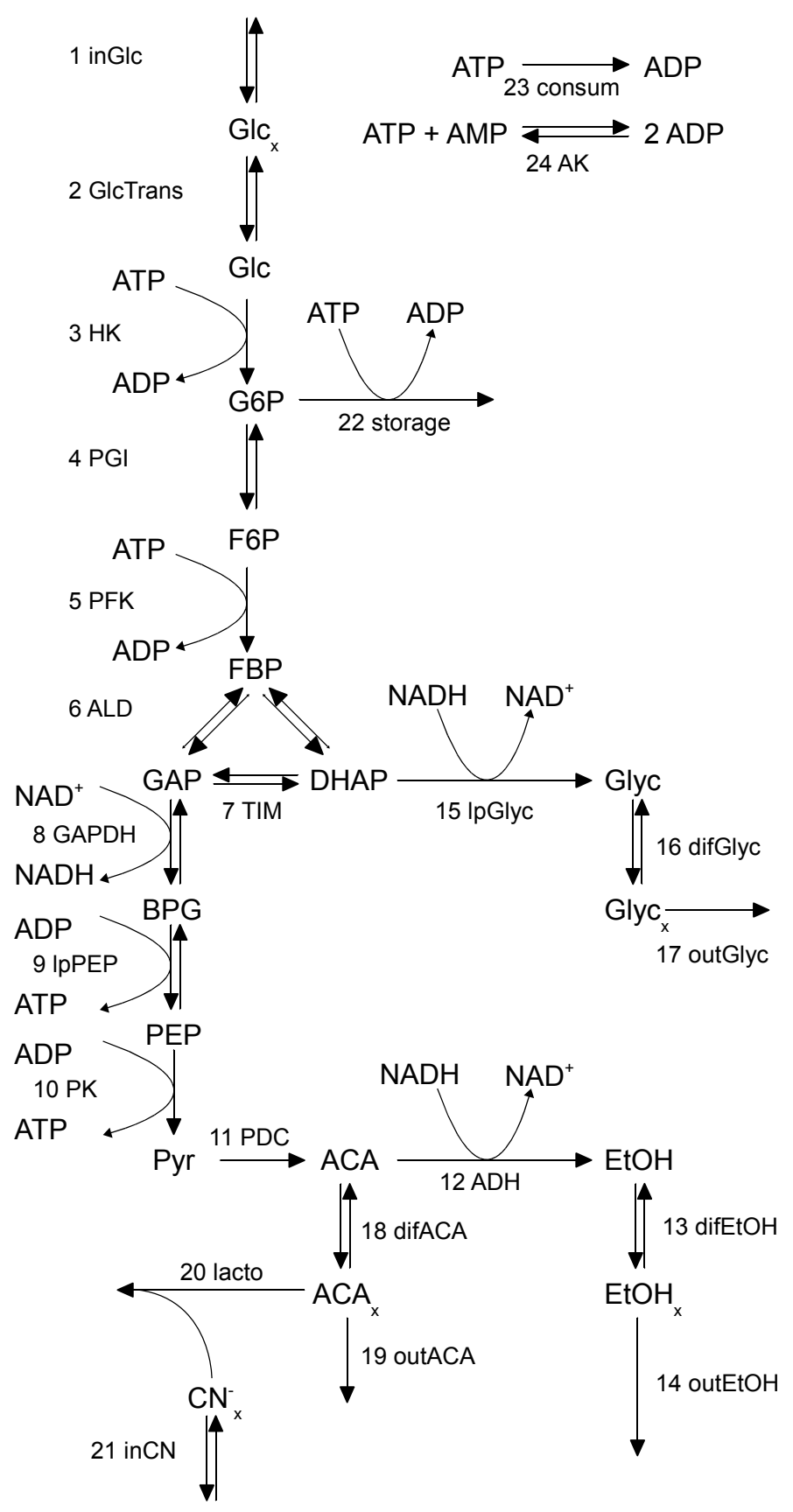

FIG. 1: Metabolic reaction network of glycolysis in yeast, adopted from Hynne et al. [20]. $x=$ extern, out=species to be export from the cellular volume, in=cellular intake.

triphosphate (ATP) to one molecule of glucose, serves as a temporary energy storage. The products of this reaction are glucose 6-phosphate $(\mathrm{G} 6 \mathrm{P})$ and adenosine diphosphate (ADP). As the cellular membrane is permeable for glucose but not for G6P, the conversion of glucose into G6P enables the cell to accumulate G6P. G6P is subsequently converted to fructose 6 -phosphate $(\mathrm{F} 6 \mathrm{P})$ by the enzyme phosphoglucoisomerase (PGI) (reaction 4) or stored, using one molecule of ATP (reaction 22). F6P is phosphorylated to fructose 1,6-bisphosphate (FBP) by 
the key enzyme phosphofructokinase (PFK) at the expense of one molecule of ATP (reaction 5). ATP is not only a substrate of this reaction, but italso eperates as an inhibitor of phosphofructokinase, which is an allosteric protein. On the other hand, AMP is an activator of PFK. This key regulatory step, the second phosphorylation, next leads to the splitting of FBP into dihydroxyacetone phosphate (DHAP) and glyceraldehyde 3-phosphate (GAP), mediated by the enzyme aldolase (ALD) (reaction 6). DHAP is transformed by triosephosphate isomerase (TIM) into GAP (reaction 7) or converted into glycerol (Glyc), using one molecule nicotinamide adenine dinucleotide (NADH) (reaction 15). GAP is oxidated by glyceraldehyde 3 -phosphate dehydrogenase (GAPDH) using one molecule of NAD and adding a phosphate group, producing 1-3-bisphosphoglycerate (BPG) (reaction 8). Each of the following two reactions produces one molecule of ATP by transferring one phosphate residue to ADP (reactions 9 and 10). During these reactions, BPG is converted into phosphoenol pyruvate (PEP), which is subsequently transformed into pyrovate (Pyr), catalyzed by pyruvate kinase $(\mathrm{PK})$. Cyanide $\left(\mathrm{CN}_{x}^{-}\right)$, which is imported into the cell (reaction 21), acts as an inhibitbor and prevents the inflow of pyruvate into the citric acid cycle. Instead, Pyr is fermented by acetaldehyde (ACA) into ethanol (EtOH) (reactions 11 and 12).

In this model, only glucose and cyanide enter the system (reactions 1 and 2), and ethanol, glycerol, and acetaldehyde diffuse through the membrane into the extracellular volume (reactions 13, 16, 18). This simplification leads to two conservation laws: The total concentrations $\mathrm{A}=\mathrm{ATP}+\mathrm{ADP}+\mathrm{AMP}$ and $\mathrm{N}=\mathrm{NADH}+\mathrm{NAD}$ remain constant.

Hynne et al. [20] built on results of extensive biochemical studies to set up the rate equations and to determine the kinetic parameters and the operating point, which was found to be located near a Hopf bifurcation. The exact location of the bifurcation point was found experimentally by using $\left[\mathrm{Glc}_{x}\right]_{0}$ as a bifurcation parameter.

This full-scale model of glycolysis in yeast provides an excellent basis for further studies of the dynamics of glycolysis. In order to obtain a deeper understanding of its design principles, we applied two different approaches: a) we analyzed the model at its stationary state by a method that focuses on the general features of the reactions and b) we integrated numerically the rate equations under the influence of extrinsic and intrinsic noise. Extrinsic noise is due to disturbances in the cell's environment [25] and is modeled as a variation of the parameters. Intrinsic noise is due to local fluctuations in the number of molecules of each chemical species and is modeled by adding random fluctuations to the concentrations, deliberately violating the perfect-mixing hypothesis.

\section{METHODS}

\section{A. Generalized method}

The generalized method, developed by Gross and Feudel [23] and adapted to metabolic networks by Steuer et al. [24], bridges the gap between structural kinetic modeling, based on the stoichiometry alone, and explicit kinetic models of metabolism [24]. By way of this method, we are able to search in a large ensemble of models for bifurcations and oscillatory regions via a local stability analysis. The approach assumes that an explicit model is not necessary to study the onset of oscillations. For example, in order to determine under which conditions a steady state loses stability, only a linear approximation to the system at this state is necessary, as one just needs to know the eigenvalues of the associated Jacobian.

Instead of focusing on a particular set of differential equations, this approach is based on the parametric representation of the Jacobian matrix, where each element has a well-defined interpretation in biological terms [24]. Once the Jacobian is constructed, the method gives a detailed account on the dynamical capabilities of a molecular network, including the stability of steady states, the possibility of sustained oscillations as well as the existence of chaotic regimes. The analysis is quantitative and therefore assesses the robustness of a system in a specified region in parameter space [24].

The approach decomposes the Jacobian $J$ for $m$ metabolites and $r$ reactions into a product of two matrices, denoted $\boldsymbol{\Lambda}$ and $\boldsymbol{\Theta}_{\boldsymbol{x}}^{\boldsymbol{\mu}}$ below, the elements of each of which have a well defined interpretation. This decomposition is obtained by starting from the set of differential equations

$$
\frac{d \boldsymbol{S}(t)}{d t}=\boldsymbol{N} \boldsymbol{\nu}(\boldsymbol{S}, \boldsymbol{k})
$$

where $\boldsymbol{S}$ and $\boldsymbol{N}$ denote the vector of metabolite concentrations and the stochiometric matrix, where $\boldsymbol{\nu}(\boldsymbol{S}, \boldsymbol{k})$ denotes the vector of reaction rates, which depend on the metabolite concentrations $\boldsymbol{S}$ and on a set of parameters $\boldsymbol{k}$. Under the assumption that there exists at least one state $\boldsymbol{S}^{0}$ fulfilling the steady-state condition

$$
\boldsymbol{N} \boldsymbol{\nu}\left(\boldsymbol{S}^{0}, \boldsymbol{k}\right)=0
$$

and using the definitions

$$
\Lambda_{i j}:=N_{i j} \frac{\nu_{j}\left(\boldsymbol{S}^{0}\right)}{S_{i}^{0}} \text { and } \mu_{j}(\boldsymbol{x}):=\frac{\nu_{j}(\boldsymbol{S})}{\nu_{j}\left(\boldsymbol{S}^{0}\right)}
$$

as well as the variable transformation

$$
x_{i}=\frac{S_{i}}{S_{i}^{0}},
$$

the Jacobian in the new variable $\boldsymbol{x}$ reads

$$
\boldsymbol{J}=\boldsymbol{\Lambda} \Theta_{\boldsymbol{x}}^{\mu} \text { with } \Theta_{\boldsymbol{x}}^{\boldsymbol{\mu}}:=\frac{\partial \boldsymbol{\mu}(\boldsymbol{x})}{\partial \boldsymbol{x}}
$$


The matrix $\Lambda$ is fully defined by the stoichiometric matrix $(\boldsymbol{N})$, a set of steady-state concentrations $\boldsymbol{S}^{0}$, and a steady state flux distribution (determined by $r-\operatorname{rank}(\boldsymbol{N})$ free parameters, i.e., elementary flux modes) $\nu^{0}$. The matrix $\Theta_{\boldsymbol{x}}^{\mu}$ defines the effective kinetic order of each reaction with respect to the metabolites.

For each element of the $r \times m$ matrix $\boldsymbol{\Theta}^{\boldsymbol{\mu}}$, we can specify a well defined interval of possible values for the elements $\boldsymbol{\Theta}_{\boldsymbol{x}}^{\boldsymbol{\mu}}$ without referring to the explicit functional form of the particular rate equation; for example $\Theta_{x}^{\mu}=1$ corresponds to a simple mass-action model, while values $\in[0,1]$ represent Michaelis-Menten kinetics (for further information see [24]).

Drawing entries of $\boldsymbol{\Theta}_{\boldsymbol{x}}^{\boldsymbol{\mu}}$ from a random distribution, generates efficiently a large ensemble of possible Jacobians for the glycolysis network. We select those models that lead to sustained oscillations, i.e., that have a positive real part of the largest eigenvalues, with complex conjugated imaginary parts. We can then compare the average steady-state values of $\boldsymbol{\Theta}_{\boldsymbol{x}}^{\boldsymbol{\mu}}$ within the subensemble of these oscillating models to the initial value in order to obtain information about the influence of each reaction on the stability of the system. A similar procedure can be performed for the metabolite concentrations and for the elementary flux modes. This procedure allows us to identify those reactions and metabolites that have a strong influence on the dynamics of glycolysis.

\section{B. Influence of Noise}

Contrary to the generalized method, which is based on the general structure of the rate equations and a local stability analysis, the second route taken in this work focuses directly on the influence of noise on the dynamics of glycolysis. Real cells do not show well-mixed intracellular and extracellular phases, rather the molecules are subject to diffusive motions through the cell, leading to cytosolic fluctuations of the metabolite concentrations. We model the dynamics and these fluctuations in the concentrations $\boldsymbol{S}$ by a stochastic differential equation, as recently discussed in [26]

$$
\dot{\boldsymbol{S}}=f(\boldsymbol{S}, \boldsymbol{k}, t)+\beta \cdot \boldsymbol{S} \cdot \boldsymbol{W}
$$

with $\boldsymbol{W}$ a (vectorial) Wiener process of (component wise) variance 1 and vanishing average. The global ratio $\beta$ gives the "noise" strength relative to the absolute concentrations. Again, $\boldsymbol{k}$ is the set of parameters of the reaction kinetics. In the following, we assume stationarity, so that $f$ in eq. (5) does not explicitly depend on time $t$. This stochastic differential equation can be solved numerically by an explicit order 1.5 Runge-Kutta method [27] with sufficient accuracy.

To evaluate the influence of noise on the dynamics of glycolysis in yeast, we will analyze the proportion of oscillating systems compared to the system without noise, the mean values and standard deviation of the metabolic concentrations, and the resulting time-averaged correlation matrix. This is repeated for several sets of slightly varied parameters $\boldsymbol{k}$ in order to account for external noise and uncertainty in these parameters, too.

\section{RESULTS}

\section{A. Generalized method}

We applied the generalized method to the full-scale model of glycolysis outlined above around the empirical operation point, which is located close to a Hopf bifurcation. With this method, the stability of a fixed point is evaluated as a function of the values of the generalized parameters. We performed three different series of evaluations, during each of which one type of parameters was varied while the others were retained at the operation point. The variation of a set of parameters was done by choosing at random each parameter value from an interval of a width of 5 percent around its value at the operation point. For each series, $10^{6}$ sets of parameters were generated, and the eigenvalues of the Jacobian were computed numerically using a GSL routine for real nonsymmetric eigensystems [28].

Then, we determined the mean values of the sampled parameters for the subset of oscillating systems and plotted the logarithm of the ratio of these values to the overall mean, similar to the procedure performed in [24]. Figures 2,3 , and 4 show the result of this analysis. A positive (negative) value means that the parameter takes a larger (smaller) value in oscillating systems than the original value of Hynne et al.

Figure 2 shows the influence of the metabolic concentrations on the occurrence of oscillations. The generalized method allows us to vary the fixed point metabolic concentrations while retaining the linear combination of elementary flux modes and all saturation parameters $\boldsymbol{\Theta}_{\boldsymbol{x}}^{\boldsymbol{\mu}}$ as in Hynne et al. This type of investigation cannot be done with other methods, where the variation of one quantity usually affects many other quantities. Metabolites that affect the oscillations most are those located in the center of the network (G6P, F6P, FBP, GAP, DHAP), together with those participating in the conservation laws (ATP, AMP, ADP, NADH, NAD). These define a core of the system that controls the dynamics. Figure 2 shows that a high concentration of ATP in the cell leads to oscillations. This can be explained by the inhibitory effect ATP has on the key enzyme PFK: a high ATP concentration results in a strong negative feedback and a slower flow through the network; both effects favor oscillations. Similarly, an increased fixed point concentration of F6P or FBP with fixed values of the saturation parameters indicates a slower flow through the system and therefore an increased tendency to oscillations.

Our findings generalize earlier studies on model reduction, which found the same metabolites as the model core of glycolysis in Saccharomyces cerevisiae [12, 21, 29, 30]. 


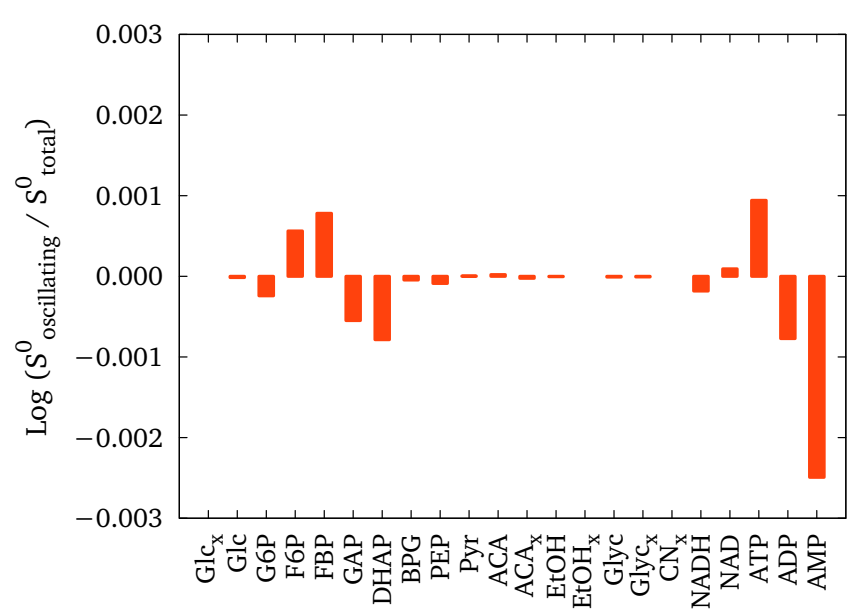

FIG. 2: Dependency of the occurrence of oscillations on the metabolic concentrations. Shown is the ratio of the mean concentrations for oscillating systems $S_{\text {oscillating to their value }}^{0}$ the operation point $S_{\text {total }}^{0}$.

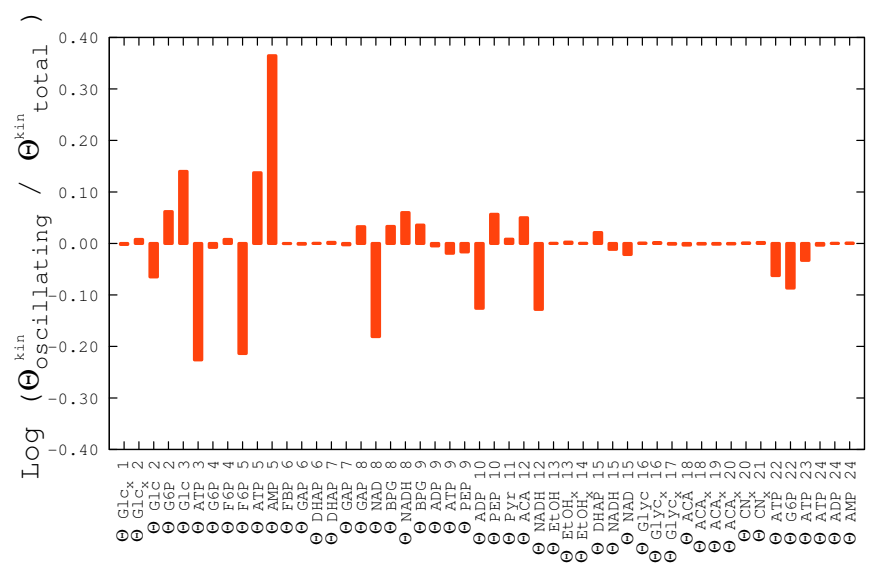

FIG. 3: Dependency of the occurrence of oscillations on the saturation parameters. Shown is the ratio of their values for oscillating systems $\Theta_{\text {oscillating }}^{0}$ to their values at the operation point $\Theta_{\text {total }}^{0}$.

Madsen et al. [12] and Schmiedel et al. [29] found also that the instability of the stationary state originates from the autocatalytic effect of ATP on its own production. A similar conclusion was obtained by Kourdis et al. [22], who showed that glycolytic oscillations cannot be understood as a simple cause-and-effect chain through the intermediates of the system.

Figure 3 shows the results for varying saturation parameters $\Theta_{x}^{\mu}$. A negative value is evidence of a parameter favoring oscillations when a related concentration is closer to saturation, i.e., when a change in the respective concentration affects the reaction rate to a lesser extent. The figure shows that reaction 5 has the strongest effect on the oscillations. In addition, reactions $3,8,12,10$,
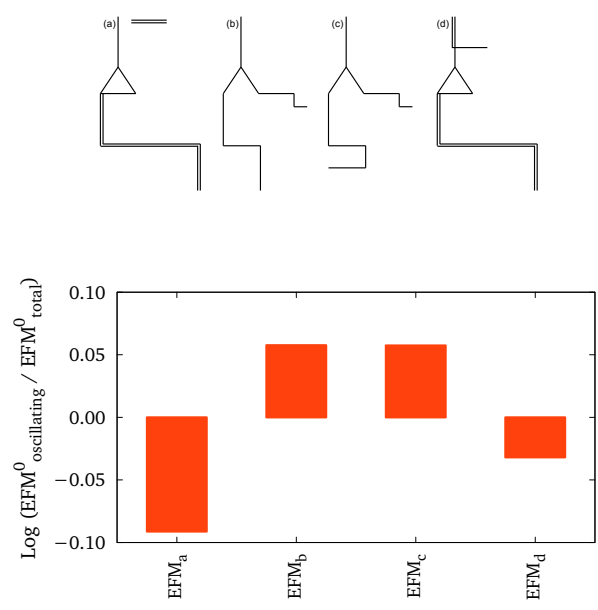

FIG. 4: Elementary fluxmodes of glycolysis and the dependency of the occurence of oscillations on linear combinations of elementary fluxmodes.

22 have a considerable effect, all of which involve either ATP or NADH. The sensitivity to the saturation with respect to Glc in reaction 2 indicates that oscillations are induced when glucose concentration itself does not affect the transport of glucose into the cell, i.e., when the transport of glucose is saturated. This is the case when little energy is used, i.e., when the flow through the system is slow.

Third, we analyzed the dependency of the oscillations on the linear combination of elementary flux modes (see Fig. 4). An elementary flux mode is a minimal set of enzymes that can operate at a steady state with all irreversible reactions proceeding in the direction implied thermodynamically. Any steady-state flux pattern can be expressed as a non-negative linear combination of these subpathways [31, 32]. The elementary flux modes of glycolysis are shown in the upper part of Fig. 4. Each subpathway must start with the entry of glucose into the cell and is determined by the conservation of internal metabolite concentrations. The possible combinations of branches are limited by the stationary condition for the conserved sums of metabolites, $\mathrm{A}=\mathrm{ATP}+\mathrm{ADP}+\mathrm{AMP}$ and $\mathrm{N}=\mathrm{NADH}+\mathrm{NAD}$ (see Hynne et al. [20]).

In oscillating models, the elementary modes $b$ and $c$ are overrepresented (see Fig. 4), i.e., those modes that include reaction 15, the production of glycerol. Elementary modes $a$ and $d$ in contrast include reaction 7 , the conversion of DHAP into a second molecule of GAP, leading to a twofold occurrence of reaction 8 and all following reactions. Therefore, elementary modes $a$ and $d$ lead to a high ATP production in the cell and are preferred when ATP demand is high and when oscillations are unlikely. In contrast, modes $b$ and $c$ lead to a high storage rate and much less ATP production. They dominate when ATP demand is low, i.e., when the flow through the system is slow and when the system tends to oscillate. 

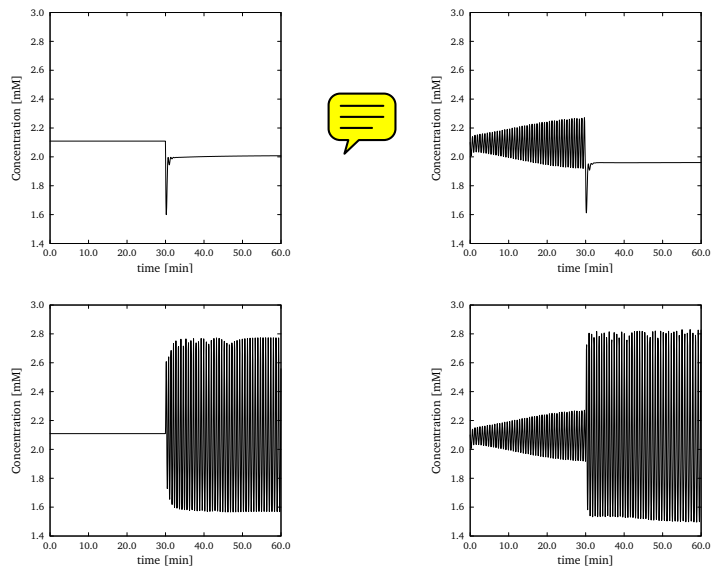

FIG. 5: ATP concentration in the stable (left column) or oscillating (right column) system with ATP consumption rate $k_{23}$ being doubled (first row) or halved (second row) after 30 minutes.

All these findings suggest that oscillations are a mere side effect of the negative feedback built into the pathway in order to keep the ATP concentration within desired limits. In order to test the extent to which the system manages to maintain the ATP level, we simulated time series by direct numerical integration of the rate equations. We determined the stationary behavior for different rates of ATP consumption (reaction 23). Figure 5 shows the effect of doubling or halving the rate of reaction 23 on a stable and an oscillating system. In the left column the system is initialized with the kinetic parameters in the stable regime (bifurcation parameter $\left[\mathrm{Glc}_{x}\right]_{0}=17$ ), and in the right column the system is initialized with the kinetic parameters in the oscillating regime $\left(\left[\mathrm{Glc}_{x}\right]_{0}=24\right.$; for other parameter values see appendix). After 30 minutes, the ATP consumption rate $k_{23}$ is either doubled (first row) or halved (second row). While a reduced consumption rate drives the system towards stronger oscillations, the mean ATP concentration changes barely. Doubling the demand reduces the ATP level only by 5 percent. Our investigations of the response of the system to noise in the next section will support this finding: the system evolved such that the mean ATP level remains constant when overall rates change.

\section{B. Influence of Noise}

We simulated 844 perturbed systems with $5 \%$ noise on the relative concentrations. Each system was assigned kinetic parameters that were chosen at random within a $5 \%$ interval around the operation point given by Hynne et al [20]. After a transient period of 100 minutes, we started to analyze a time series. These analyses gave the correlation matrix, the proportion of oscillating systems, as well as the means and standard deviations of the metabolite concentrations.

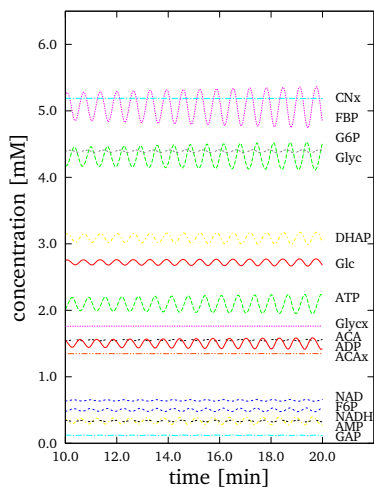

(a)



(b)
FIG. 6: (a) Oscillations in the deterministic system and (b) the system under the influence of noise.

Figure 6 exemplifies time series of a system with and without noise. The oscillations in the system without noise have a fixed phase relation among the different metabolites. G6P and F6P are in opposite phase to FBP, i.e., metabolites before and after reaction 5 are out of phase. Because of the conservation of the sum of ATP, $\mathrm{ADP}$ and AMP, two of the three metabolites oscillate in phase, whereas the third must show the opposite phase. Due to conservation of the sum of NADH and NAD, these two metabolites have opposing phases.

Correlations between metabolites are shaped by a combination of stoichiometric and kinetic effects [33]. One would expect a high correlation of metabolites directly connected to each other. However, it has been shown [34] that this assumption does not need to hold in all cases, because the variance in enzyme concentrations affects the metabolites by equal amounts, but in different directions. In contrast, metabolites having no direct connection can show a high correlation. Camacho et al. [34] tried to reveal the underlying mechanisms and found that metabolites in chemical equilibrium have a nearly perfect positive correlation, leading to the assertion that negatively correlated metabolites are out of equilibrium. Moderate correlations between metabolites suggest a "hidden causality", namely a shared enzyme. Another factor affecting correlations is mass conservation, which is mandatory in metabolism; common examples are NAD/NADH and ATP/ADP/AMP.

We quantify the correlation $C_{i j}$ of substance $i$ and $j$ in the simulated time series by computing the instantaneous cross-correlation [35] of their respective concentrations $c_{i}$ and $c_{j}$ :

$$
C_{i j}:=\frac{1}{T_{\max }} \sum_{t=1}^{T_{\max }} c_{i}(t) \cdot c_{j}(t) .
$$

In Fig. 7, the correlation matrix for the noisy system shows strong correlations of extracellular metabolites and strong correlations between input $\left(\mathrm{Glc}_{x}\right)$ and output 


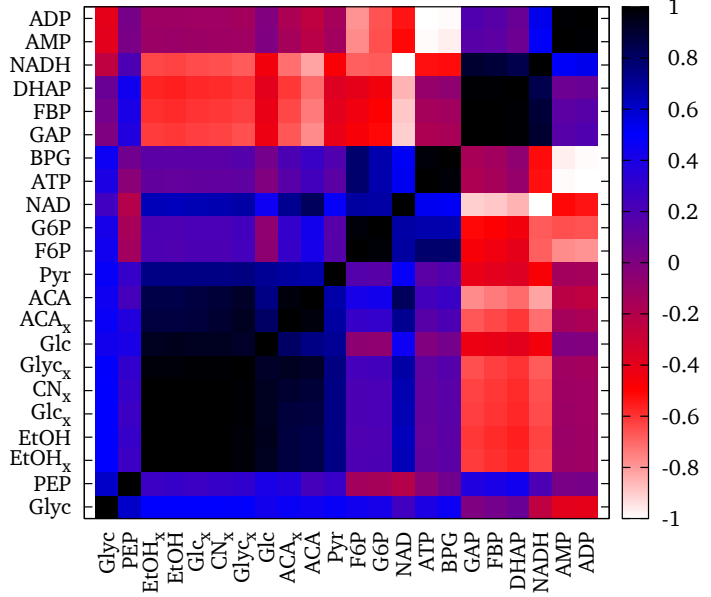

FIG. 7: Correlations of metabolites' mean values.

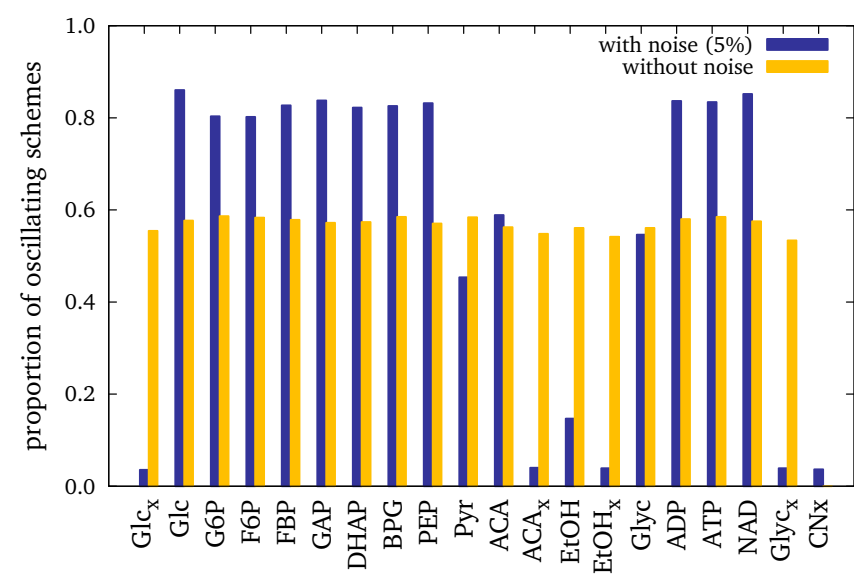

FIG. 8: Proportion of oscillating systems of the 844 simulations.

(EtOH, ACA, Glyc and the extracellular forms, respectively). As can be seen in Fig. 6, extracellular metabolites do not show oscillations. Then, high correlations arise due to a simple mechanism: more input into the system generates more output. Furthermore, the three metabolites FBP, GAP, and DHAP that form the central triangle in the network are highly correlated, because their reactions are fast. Those metabolites located next to each other in the network and associated with the conversion of ATP into ADP or NADH into NAD or the reverse reactions, are correlated negatively. Additionally we found anticorrelations between ATP and ADP/AMP and between NAD and NADH.

Using a Fast Fourier Transform analyis, we evaluated in how many of the 844 systems metabolites show oscillations. The fourier spectrum was computed based on a sampling frequency of $8 \frac{1}{3} \mathrm{~Hz}$. The data was smoothened by averaging over 30 adjacent data points. We deter- mined whether the spectrum shows a maximum in the sampling range of $[1 / 2 ; 1] \mathrm{min}^{-1}$, which contains the oscillation period of $\approx 40 \mathrm{sec}$ of the original system. This approach allows to understand potential (resonance) frequency shifts due to noise. Figure 8 shows the fraction of systems in which each metabolite oscillates in the noisy simulations. For comparison, the corresponding proportion in the noise-free system is also shown (with the same internal parameters). When noise is added, the proportion of oscillating systems increases by some 30 percent, as can be seen for the metabolites PEP, F6P, BPG, FBP, ATP, ADP, GAP, DHAP, G6P, and NAD. The extracellular metabolites $\mathrm{Glc}_{x}, \mathrm{ACA}_{x}$, Glyc $x$ and EtOHx oscillate in fewer systems when noise is added.

In the absence of noise and at the operation point determined by Hynne et al., the concentrations of $\mathrm{CN}_{x}$, $\mathrm{Glyc}_{x}, \mathrm{ACA}_{x}$, and GAP do not fluctuate. $\mathrm{CN}_{x}$ oscillates only in very few systems in the absence of noise.

The finding that the core metabolites are more prone to oscillating when noise is added, is in agreement with the general observation that white noise can induce sustained oscillations when a system is near a Hopf bifurcation and would show a dampened oscillation in absence of noise. This is a resonance effect [36].

A potential explanation for the loss of oscillations in the external metabolites is the fact that they accumulate the noise of the previous reaction steps, and therefore the random fluctuations become so large that the signatures of oscillations are lost due to a bad signal-to-noise ratio.

Next, we investigate which kinetic parameters affect the occurrence of oscillations in the noisy set-up. We call a system "oscillating" if NADH shows oscillations. We choose NADH as a reference since experimental data are almost exclusively determined in relation to the NADH concentration. We compare the mean of the parameters of the oscillating systems with the respective mean of all simulated systems and calculate the logarithm of this ratio. Figure 9 shows the result of this analysis. A positive (negative) value indicates that the parameter takes a larger (smaller) value in oscillating systems than the original value of Hynne et al.

In contrast to Fig. 3, many reactions affect the oscillations equally strongly, and reaction 5 is not the dominant one. We explain this difference by the fact that the parameters are varied differently in Fig. 3 than in Fig. 9. In Fig. 3, the saturation parameters were varied for fixed metabolite concentrations - this can only be realized by a simultaneous variation of the reaction constants.

We conclude from these results that while the saturation parameters of reaction 5 are the main determinants of the oscillations, these saturation parameters depend on several kinetic parameters of the original model. Therefore, a variation of each of these parameters can induce oscillations. This explanation resolves the contradiction between the conclusions by Bier et al. [16] and those by Goldbeter [14] and Sel'kov [15] mentioned in the Introduction.

Next, we evaluated the robustness of the reaction net- 




FIG. 9: Influence of individual parameters in the system with noise on the occurence of oscillations.

work with respect to noise. From a bio-engineering point of view, important measures of robustness are the mean values of concentrations and their standard deviations.

Figure 10 shows the means and time-course standard deviations of all metabolites from our 844 simulations. For reference, we show also the original values from Hynne et al. The reference line of $5 \%$ is a first approximation to the order of magnitude of the standard deviation. It would be exactly realized if all metabolites were uncoupled. However, the reaction network topology and the enzyme kinetics increase the magnitude of fluctuations in some concentrations, while suppressing such fluctuations in others: a striking feature is the small fluctuation in ATP, which indicates that in oscillating systems and in stable systems the mean ATP level remains constant for most if not all 844 simulations.

Other metabolites with small error bars are ADP, AMP, NAD and NADH, which are also related to the cellular energy supply. This indicates the importance of a stable amount of energy, but can additionally be induced by conservation laws. The network appears to have been evolved such that the ATP level is kept at an optimum level. Even with noise, the system manages to keep the energy level constant, i.e., reaction 5 ensures that the remaining steps of glycolysis are adjusted to the level of ATP. One mechanism for achieving a constant ATP level is the interception of fluctuations by an internal buffer, such as FBP: In the systems with noise, FBP is the only internal metabolite that has a standard deviation larger than the noise level, and a considerable deviation of its mean from the value at the operation point.

FBP is highly correlated with DHAP and GAP, i.e., it affects reaction 6 (see Fig. 1). If FBP accumulates in the cell, reaction 6 runs faster, leading to fluctuations in the external metabolites. Indeed, the metabolites that show a significant standard deviation and a shift of their mean value are $\mathrm{EtOH}, \mathrm{EtOH}_{x}$, and $\mathrm{Glc}_{x}$. In addition, Glc has
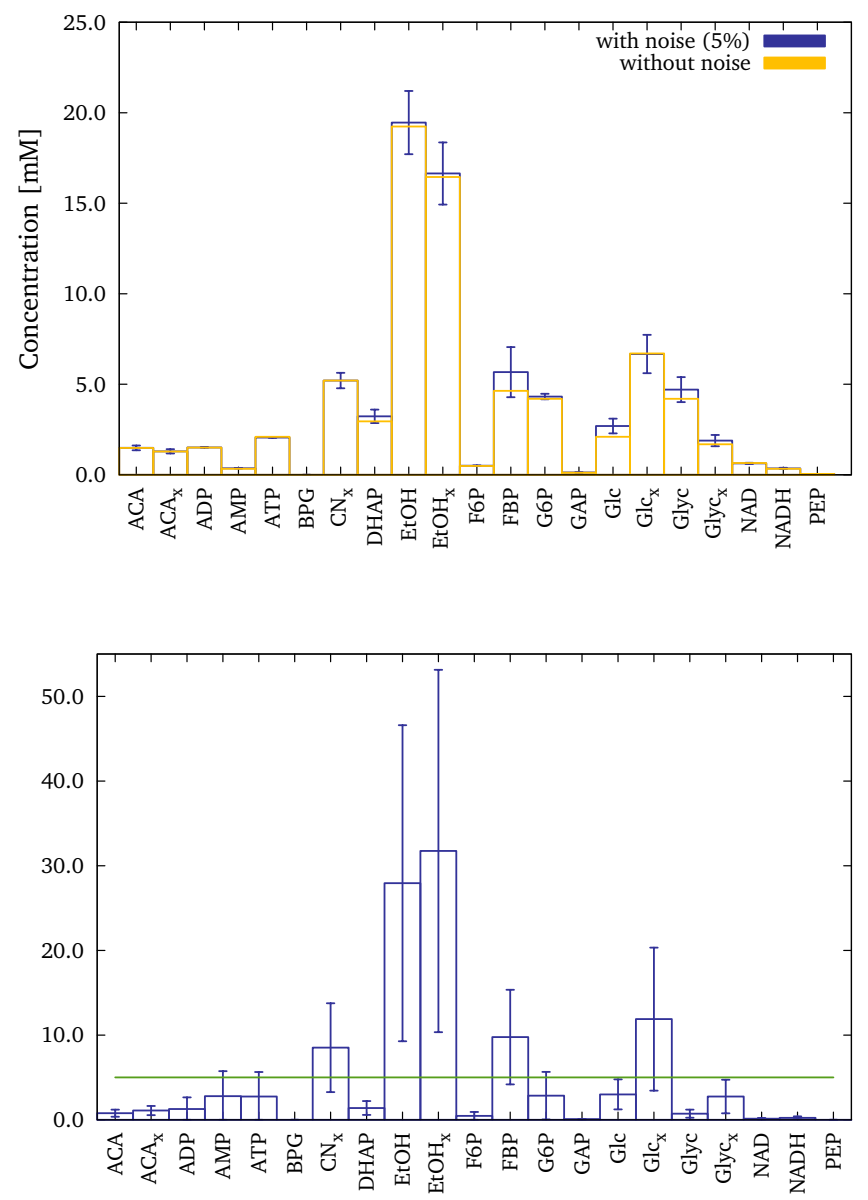

FIG. 10: Mean and standard deviation of all metabolites analyzed for the 844 simulations. Top: Mean concentrations and the original mean values from Hynne et al. [20] are shown. Error bars are standard deviations from sampling of the 844 different replica. Bottom: Standard deviation in the time series of metabolic concentrations. Again, error bars are standard deviations from the sampling over the 844 parameter choices. For reference, we show also the corresponding $5 \%$ noise level imposed on the metabolites' concentrations (green line).

a shift of its mean value, and $\mathrm{CN}_{x}$ has a large standard deviation. All these metabolites are at the boundary of the network. There is no need to regulate the values of their concentrations, and in any case no such regulation is built into the model by Hynne et al. The reason why Glyc and $\mathrm{Glyc}_{x}$ as external metabolites do not have large fluctuations lies in the very small reaction rate of diffusion reaction 16. The export of Glyc is very slow, so that fluctuations in the interior of the cell do not affect this reaction. 


\section{DISCUSSION AND CONCLUSIONS}

In this paper, we have investigated the susceptibility of the full-scale model for glycolysis to internal and external noise. We applied a diverse set of tools and approaches, in particular generalized models and numerical integration of the stochastic differential equations. For both approaches, we generated an ensemble of systems subject to an external noise of 5 percent on the kinetic parameters.

The generalized method revealed that the saturation of the PFK-reaction has the strongest influence on the occurrence of oscillations. This reaction is catalyzed by phosphofructokinase and inhibited by ATP; it introduces a negative feedback into the process of glycolysis. When the original kinetic parameters are varied instead of the saturation parameters, many reactions appear to affect the occurrence of oscillations. Due to its generic nature which takes into account only those effective quantities that affect the eigenvalues of the stability matrix, the method of generalized models can thus identify the general features of a model that are relevant for a certain type of dynamical behavior much more clearly than the standard approaches based on the original parameter sets. In fact, this is the main difference between the generalized models and metabolic control analysis, which is also based on a linear stability analysis of stationary points. If the exact functional form of the chemical reactions involved in the different reaction steps is not precisely known, the generalized method has the advantage that it only relies on a few generic features.

Our investigations revealed that the strong negative feedback and the longer time delay that are present at higher ATP concentrations are the mechanism that causes the oscillations. This situation manifests itself also in a predominance of those metabolic pathways that produce less ATP, as revealed by studying the influence of the different elementary flux modes on the occurrence of oscillations.

Since oscillations occur when a significant amount of ATP is present and the flow through the network is slow, they cannot be the result of an optimization of the network for maximum yield or maximum rate. Our study rather suggests that the oscillations are a side effect of other design principles of the process of glycolysis. However, oscillations can be exploited by the cell for other purposes, such as intracellular and intercellular communication and regulatory functions [37]. Oscillations carry the information that there is plenty of energy supply. Indeed, glycolytic oscillations were observed to be synchronized, implying the coupling of the metabolism between different cells. Richard et al. [38] and Bier et al. [39] conclude that cells can influence each other via a shared concentration of ACA, which is involved in the conserved NAD/NADH cycle. They state that in the case of fast synchronizing processes the cells would immediately synchronize their ATP concentrations and consequently their oscillations. But due to its slow dynamics relative to the glycolytic oscillations, the synchronization might be a driving force towards equalizing the ATP concentrations. In the model by Hynne et al., synchronization of all cells is assumed [20], and this might be the ultimate cause of the constant level of ATP.

Our numerical simulation of the model with noise supports the hypothesis that the glycolysis pathway evolved such that it operates robustly: in particular, the mean ATP level in the stationary state changes very little when the ATP consumption rate changes by a large amount or when extrinsic or intrinsic noise is added to the system. The phase correlations that are present in oscillating systems in the absence of noise remain the same in the presence of noise. Our findings thus complement and extend the findings by Buzi et al. [18] that the region of attraction of the fixed point at which the process operates is large. We also found that while the concentrations of most internal metabolites have small standard deviations in the presence of noise, the metabolites at the boundary of the network appear to accumulate the fluctuations.

While the stationary ATP level is very robust, it can nevertheless change transiently, as reported by the authors of [40], who measured the ATP level in cells that had been starved and were then given a certain amount of glucose. In this experimental setup, the ATP level declined after addition of glucose until the glucose reservoir was depleted, and then increased again.

To conclude, our synergistic methodology based on a generalized method and on numerical integration in the presence of noise of a full-scale model of glycolysis, provides strong evidence that the design principle of this network is robustness and that oscillations are a side effect of the negative feedback that is required to achieve this robustness.

\section{ACKNOWLEDGMENTS}

$\mathrm{KH}$ is grateful for financial support by the Fonds der chemischen Industrie and the Freunde der TU Darmstadt. EG and BD acknowledge support from the Deutsche Forschungsgemeinschaft under grant number Dr300/4-2. 
VII. APPENDIX

Tables I, II and III show the metabolic concentrations, the values of the kinetic parameters and the rate equations in the model.

\begin{tabular}{|c|c|c|}
\hline Metabolite & $S_{\text {stable }}^{0}[m M]$ & $S_{\text {oscillating }}^{0}[m M]$ \\
\hline $\mathrm{Glc}_{x}$ & 0.8559 & 6.6700 \\
\hline Glc & 0.2074 & 2.7200 \\
\hline G6P & 3.9858 & 4.2000 \\
\hline F6P & 0.4671 & 0.4900 \\
\hline FBP & 3.9281 & 4.6400 \\
\hline GAP & 0.1060 & 0.1150 \\
\hline DHAP & 2.7036 & 2.9500 \\
\hline $\mathrm{BPG}$ & 0.0003 & 0.0002 \\
\hline PEP & 0.0379 & 0.0410 \\
\hline Pyr & 3.6556 & 22.1500 \\
\hline $\mathrm{CN}_{x}$ & 5.2368 & 5.1900 \\
\hline $\mathrm{ACA}$ & 1.3477 & 1.5500 \\
\hline $\mathrm{ACA}_{x}$ & 1.1718 & 1.3490 \\
\hline $\mathrm{EtOH}$ & 18.4863 & 19.5600 \\
\hline $\mathrm{EtOH}_{x}$ & 15.8094 & 16.7470 \\
\hline Glyc & 3.8219 & 4.3800 \\
\hline $\mathrm{Glyc}_{x}$ & 1.6848 & 6.6700 \\
\hline $\mathrm{ADP}$ & 1.4945 & 1.5000 \\
\hline ATP & 2.1094 & 2.1000 \\
\hline AMP & 0.3300 & 0.3300 \\
\hline $\mathrm{NAD}^{+}$ & 0.6617 & 0.6500 \\
\hline NADH & 0.3300 & 0.3300 \\
\hline
\end{tabular}

TABLE I: Metabolites' concentrations used for simulations of the stable system (bifurcation parameter $\left[\mathrm{Glc}_{x}\right]_{0}=17$ ) and for the oscillating system $\left(\left[\mathrm{Glc}_{x}\right]_{0}=24\right)[20]$.



TABLE II: Rate constants, maximum velocities $\mathrm{V}_{m}$ and parameters of glycolysis in Saccharomyces cerevisiae [20]. 


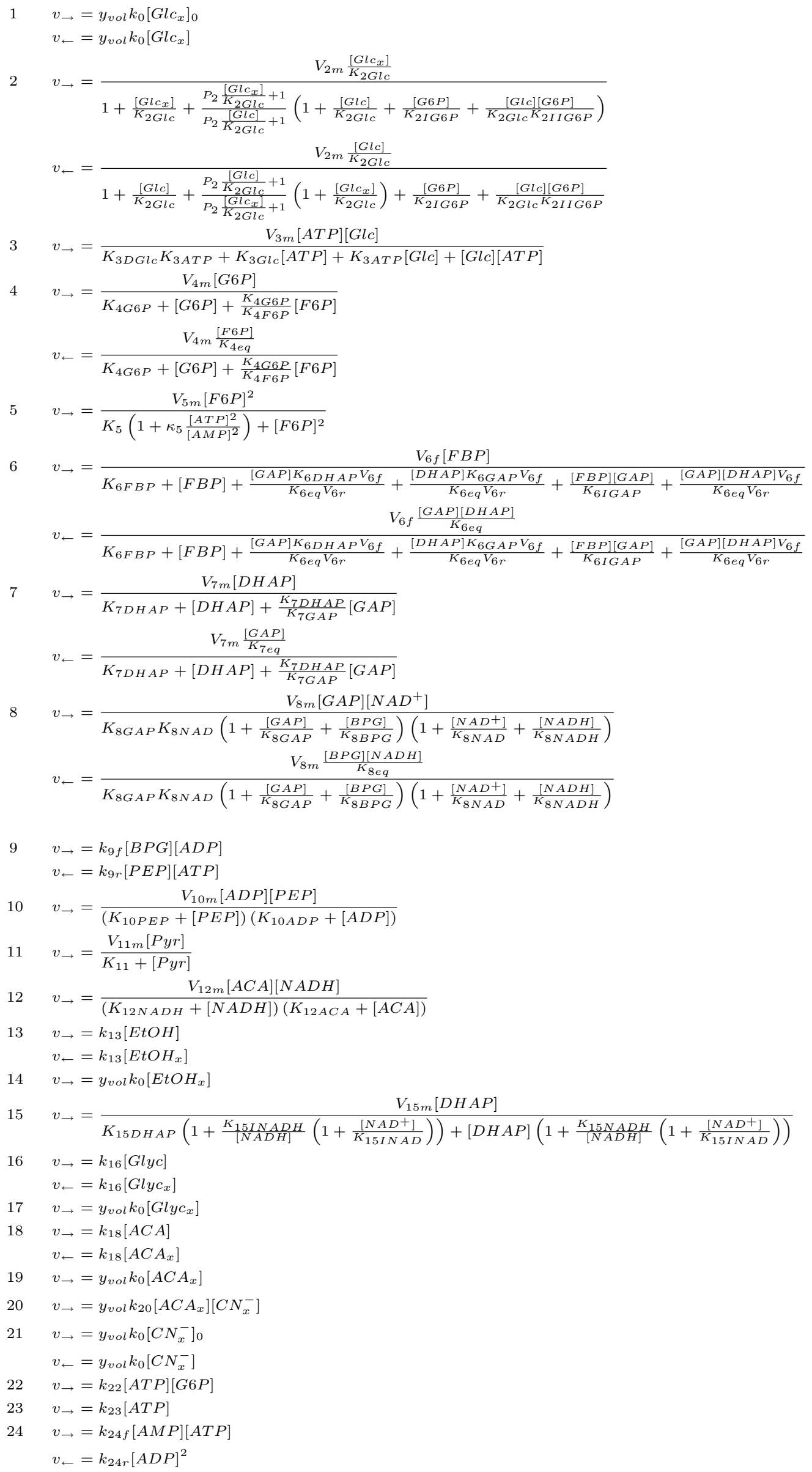

TABLE III: Rate equations in the model of glycolysis in Saccharomyces cerevisiae [20]. 
[1] T. Pfeiffer, S. Schuster, and S. Bonhoeffer, Science 292, 504 (2001).

[2] T. Pfeiffer and S. Bonhoeffer, Zeitschrift für Physikalische Chemie 216, 51 (2002).

[3] J. Nielsen, Mol. Syst. Biol. 3 (2007).

[4] S. Schuster, T. Pfeiffer, and D. A. Fell, J. Theor. Biol. 252, 497 (2008).

[5] A. Stephani and R. Heinrich, B. Math. Biol. 60, 505 (1998).

[6] F. A. Chandra, G. Buzi, and J. C. Doyle, Proceedings of Americal Control Conference pp. 319-324 (2009).

[7] B. Hess and K. Brand, Helgoland Marine Research 14, 129 (1966).

[8] P. H. Richter and J. Ross, Science 211 (1981).

[9] B. Teusink, C. Larsson, J. Diderich, P. Richard, K. van Dam, L. Gustafsson, and H. V. Westerhoff, J. Biol. Chem. 271, 24442 (1996).

[10] K. Nielsen, P. G. Sørensen, F. Hynne, and H.-G. Busse, Biophys. Chem. 72, 49 (1998).

[11] L. Pritchard and D. B. Kell, Eur. J. Biochem. 269, 3894 (2002).

[12] M. F. Madsen, S. Dan $\varnothing$, and P. G. Sørensen, FEBS J. 272, 2648 (2005).

[13] A. S. Silva and Y. J. A., Genet. Mol. Res. 5, 525 (2006).

[14] A. Goldbeter, FEBS Lett. 43, 327 (1974).

[15] E. Sel'kov, Eur. J. Biochem. 4, 79 (1967).

[16] M. Bier, B. Teusink, B. N. Kholodenkoo, and H. V. Westerhoff, Biophys. Chem. 62, 15 (1996).

[17] O. Wolkenhauer and M. Ullah, Systems Biology, F.C. Boogerd, F.J. Bruggeman, J.-H.S. Hofmeyr and H.V. Westerhoff (eds.) pp. 163-179 (2007).

[18] G. Buzi, U. Topcu, and J. Doyle, American Control Conference (ACC), 2010 (2010).

[19] M. Rizzi, U. Theobald, E. Querfurth, T. Rohrhirsch, M. Baltes, and M. Reuss, Biotechnol. Bioeng. 49, 316 (1996).

[20] F. Hynne, S. Danø, and P. G. Sørensen, Biophys. Chem. 94, 121 (2001).

[21] S. Danø, M. F. Madsen, H. Schmidt, and G. Cedersund, FEBS J. 273, 4862 (2006).
[22] P. D. Kourdis, R. Steuer, and D. A. Goussis, Physica D 2010, 1798 (239).

[23] T. Gross and U. Feudel, Phys. Rev. E 73, 016205 (pages 14) (2006).

[24] R. Steuer, T. Gross, J. Selbig, and B. Blasius, Proc. Natl. Acad. Sci. USA 103, 11868 (2006).

[25] M. Scott, B. Ingalls, and M. Kærn, Chaos 16, 026107 (2006).

[26] M. C. Mackey, M. Tyran-Kaminska, and R. Yvinec, J. Theo. Biol. 274, 84 (2011).

[27] P. E. Kloeden and E. Platen, Numerical Solution of Stochastic Differential Equations (Springer, Berlin, 1992).

[28] GSL GNU Scientific Library, URL http://www . gnu .org/ software/gsl/.

[29] J. Schmiedel and R. Steuer, Proceedings of the 5th Workshop on Computation of Biochemical Pathways and Genetic Networks, Heidelberg (2008).

[30] J. Wolf, J. Passarge, O. Somsen, J. L. Snoep, R. Heinrich, and H. Westerhoff, Biophys. J. 78, 1145 (2000).

[31] S. Schuster and C. Hilgetag, J. Biol. Syst. 2, 165 (1994).

[32] S. Schuster, T. Dandekar, and D. A. Fell, Trends Biotechnol. 17, 53 (1999).

[33] R. Steuer, J. Kurths, O. Fiehn, and W. Weckwerth, Bioinformatics 19, 1019 (2003).

[34] D. Camacho, A. de la Fuente, and P. Mendes, Metabolomics 1, 53 (2005).

[35] W.H. Press et al, Numerical Recipies in $C$ (Cambridge University Press, Cambridge, 1995).

[36] A. McKane, J. Nagy, T. Newman, and M. Stefanini, J. Stat. Phys. 128, 165 (2007).

[37] Y. Termonia and J. Ross, Proc. Natl. Acad. Sci. USA 78, 2952 (1981).

[38] P. Richard, B. M. Bakker, B. Teusink, K. Van Dam, and H. V. Westerhoff, Eur. J. Biochem. 235, 238 (1996).

[39] M. Bier, B. M. Bakker, and H. V. Westerhoff, Biophys. J. 78, 1087 (2000).

[40] V. C. Özalp, T. R. Pedersen, L. J. Nielsen, and L. F. Olsen, J. Biol. Chem. 285, 37579 (2010). 